\title{
BMJ Open Communication, Coordination, and Security for People with Multiple Sclerosis (COCOS-MS): a randomised phase II clinical trial protocol
}

\begin{abstract}
Heidrun Golla (D , ${ }^{1}$ Kim Dillen, ${ }^{1}$ Martin Hellmich, ${ }^{2}$ Thomas Dojan, ${ }^{1}$ Solveig Ungeheuer, ${ }^{1}$ Petra Schmalz, ${ }^{3}$ Angelika Staß, ${ }^{3}$ Vanessa Mildenberger, ${ }^{4}$ Yasemin Goereci, ${ }^{5}$ Veronika Dunkl, ${ }^{1}$ Julia Strupp (1) , ${ }^{1}$ Gereon R Fink, ${ }^{5,6}$ Raymond Voltz, ${ }^{1,3,7,8}$ Stephanie Stock, ${ }^{4}$ Oliver Cornely, ${ }^{3}$ Alexander Stahmann, ${ }^{9}$ Anne Müller, ${ }^{1}$ Peter Löcherbach, ${ }^{10}$ Lothar Burghaus, ${ }^{11}$ Volker Limmroth, ${ }^{12}$ Eckhard Bonmann, ${ }^{12}$ Kathrin Gerbershagen, ${ }^{12}$ Gereon Nelles, ${ }^{13}$ Thomas Joist, ${ }^{14}$ Judith Haas, ${ }^{15}$ Herbert Temmes, ${ }^{15}$ Clemens Warnke (iD ${ }^{5}$
\end{abstract}

To cite: Golla H, Dillen K, Hellmich M, et al.

Communication, Coordination, and Security for People with Multiple Sclerosis (COCOSMS): a randomised phase II clinical trial protocol. BMJ Open 2022;12:e049300. doi:10.1136/ bmjopen-2021-049300

- Prepublication history and additional supplemental material for this paper are available online. To view these files, please visit the journal online (http://dx.doi.org/10.1136/ bmjopen-2021-049300).

Received 27 January 2021 Accepted 29 0ctober 2021
Check for updates

(C) Author(s) (or their employer(s)) 2022. Re-use permitted under CC BY-NC. No commercial re-use. See rights and permissions. Published by BMJ.

For numbered affiliations see end of article.

Correspondence to Professor Heidrun Golla; heidrun.golla@uk-koeln.de

\section{ABSTRACT}

Introduction Patients with multiple sclerosis (MS) have complex needs that range from organising one's everyday life to measures of disease-specific therapy monitoring to palliative care. Patients with MS are likely to depend on multiple healthcare providers and various authorities, which are often difficult to coordinate. Thus, they will probably benefit from comprehensive cross-sectoral coordination of services provided by care and case management (CCM). Though studies have shown that case management improves quality of life $(\mathrm{Q} O \mathrm{~L})$, functional status and reduces service use, such benefits have not yet been investigated in severely affected patients with MS. In this explorative phase II clinical trial, we evaluated a CCM with long-term, cross-sectoral and outreaching services and, in addition, considered the unit of care (patients and caregivers).

Methods and analysis Eighty patients with MS and their caregivers will be randomly assigned to either the control (standard care) or the intervention group (standard care plus CCM (for 12 months)). Regular data assessments will be done at baseline and then at 3-month intervals. As primary outcome, we will evaluate patients' QoL. Secondary outcomes are patients' treatment-related risk perception, palliative care needs, anxiety/depression, use of healthcare services, caregivers' burden and QoL, meeting patients' and caregivers' needs, and evaluating the CCM intervention. We will also evaluate CCM through individual interviews and focus groups. The sample size calculation is based on a standardised effect of 0.5 , and one baseline and four follow-up assessments (with correlation 0.5). Linear mixed models for repeated measures will be applied to analyse changes in quantitative outcomes over time. Multiple imputation approaches are taken to assess the robustness of the results. The explorative approach (phase II clinical trial) with embedded qualitative research will allow for the development of a final design for a confirmative phase III trial.
Strengths and limitations of this study

- Patients and their caregivers will be studied over 15 months including one follow-up which allows for evaluation of sustainability and assessment of potential aftereffects.

- As we assume that care and case management is especially useful in patients with complex neurological disease, we selected patients with a higher degree of multiple sclerosis-related disability and chronic progressive disease, higher risk for treatment-related adverse effects or higher age, factors that might negatively impact the benefit-risk ratio of modern immunotherapy.

- Permission of proxy assessments will reduce missing data; however, this might potentially introduce bias.

- Blinding of the outcome assessor depends on the discretion of study participants, which might not always be feasible.

- The planned sample size (80 patients in total) in 3 strata (13-14 per stratum and treatment arm) may be too small to sufficiently estimate all relevant aspects of the proposed model for longitudinal data, particularly in all subgroups of interest.

Ethics and dissemination The trial will be conducted under the Declaration of Helsinki and has been approved by the Ethics Commission of Cologne University's Faculty of Medicine. Trial results will be published in an openaccess scientific journal and presented at conferences. Trial registration number German Register for Clinical Studies (DRKS) (DRKS00022771).

\section{INTRODUCTION}

Patients suffering from chronic neurological diseases including multiple sclerosis (MS) have complex needs, which vary, depending 
on age and degree of disability and range from organising one's everyday life to measures of disease-specific therapy monitoring and palliative and hospice care. ${ }^{12}$ Patients and their caregivers (ie, relatives or closely related persons, directly involved in the patients' care on whom the increasing care dependency can be a growing burden) often feel overwhelmed by the multitude of processes to be coordinated as they may lack necessary resources or specific knowledge. ${ }^{1-3}$ Due to their illness, patients with MS are restricted in their lives as the illness often commences in early adulthood and has significant sociomedical consequences. They have to reorient themselves professionally and in their private lives and develop physical and psychological coping strategies. We previously identified continuous communication with care coordinators who navigate severely affected patients with MS and their caregivers through challenging tasks as a strong unmet need. ${ }^{45}$ During their disease, many patients with MS depend on multiple healthcare providers, pension insurance companies and various other authorities, all of which are difficult to coordinate. We hypothesise that severely affected patients with MS will benefit from comprehensive coordination of services in which a care and case manager (CCM) assesses changing needs, eliminates fragmented care and arranges for new services to be provided. ${ }^{6}$

\section{Rationale}

Patients with MS are prototypical for neurological patients with a complex, chronic neurological disease having a high need for cross-sectoral and interdisciplinary communication, coordination and treatment-related safety management. As the number of complex neurological patients increases due to medical progress (increased longevity) and the ageing population, the evaluation of a comprehensive coordinating service for such patient populations is warranted. Making the existing healthcare and social structures more effective and easier accessible, reshaping them to meet the patients' individual needs, and identifying and addressing gaps in the current care will likely improve the patients' care. This combination of individual and superordinate coordination and development processes is the core task of CCM. The current study investigates a continuous cross-sectoral outreaching patient advocacy CCM for severely affected patients with MS and their relatives (unit of care) and compares this to standard care within existing healthcare structures. We hypothesise that the more complex and severe the disease and the more cross-sectoral the CCM, the larger the patients' and caregivers' benefit. ${ }^{7-15}$

We expect significant improvements on various levels through CCM evaluated in this study:

1) Patients: definition and determination of unmet needs (eg, disease-related, social, psychological, financial) as well as development of a resource-oriented plan of action that promotes the patient's independence and self-efficacy and provides support to relieve patients of otherwise overwhelming tasks. The CCM represents the patient's interests and works cross-sectoral and continuously over 12 months, thus acting as a reliable contact person assessing and monitoring the changing needs of patients and caregivers. A predominant focus is the comprehensive coordination of services across the continuum of care (eg, co-therapists, medical specialists). Even if these aforementioned services are no longer necessary, the CCM will continue to act as a superordinate contact person.

2) Caregivers: consequence of an increasing care dependency is a growing burden and strain for caregivers affecting various aspects of their lives. The CCM will also try to alleviate the caregiver's burden by finding possible solutions and developing coping strategies.

3) Healthcare providers and healthcare system: the CCM eliminates fragmented care, arranges for transparent and superordinate information transfer between involved healthcare providers and services, and arranges for all necessary services to be provided-existent and otherwise aiming for initiation of new healthcare services—relieving the medical specialist of this task. A portfolio will also be created, listing all suitable health service providers that will fit the patients' needs and deliver the best possible care to this patient group and their caregivers.

4) Society: a CCM as proposed in our trial is expected to save resources by a more targeted and better coordinated use of sociomedical services. This might, inter alia, lead to a reduction in the number of physician and emergency department visits or early identification of risks and potential for improvement. ${ }^{7}$

\section{Objectives}

This trial's primary objective is to examine the effects of a comprehensive cross-sectoral, long-term patient advocacy CCM for severely affected patients with MS with complex needs and their caregivers on patients' quality of life (QoL). MS significantly affects QoL, interfering with a person's ability to work, leisure activities and everyday life. Previous research has shown substantial improvements in QoL by introduction of case management (CM) into the care of patients with chronic neurological $^{101617}$ and other diseases. ${ }^{78101118-20}$

Secondary objectives include patients' (1) treatmentrelated risk perception, (2) palliative care needs, (3) anxiety and depression, (4) use of healthcare services, caregivers', (5) burden and (6) QoL, (7) meeting patients' and caregivers' needs through CCM and (8) evaluation of the CCM intervention.

To aid in the interpretative process and evaluation of the CCM intervention, we will also conduct individual semistructured in-depth interviews with a subset of patients and caregivers as well as focus groups or individual in-depth interviews with healthcare professionals.

\section{METHODS AND ANALYSIS}

\section{Trial design}

The trial is a single-centre, phase II, two arms, randomised, controlled, rater-blinded, parallel-group clinical trial 
evaluating the effects of a cross-sectoral, long-term patient advocacy CCM for severely affected patients with MS with complex needs and their caregivers to improve patients' QoL.

The trial is conducted at the Department of Palliative Medicine and the Department of Neurology of the University Hospital of Cologne and has begun in August 2020 (with the preparation phase) for the duration of 40 months (planned trial end: December 2023). Patient inclusion is scheduled to start in April 2021.

In preparation for this protocol, we have used the Standard Protocol Items: Recommendations for Interventional Trials reporting guidelines. ${ }^{21}$

\section{Recruitment}

Patients and caregivers will be recruited at the University Hospital of Cologne. Moreover, we rely on our partner institutions/organisations including neurologists (inpatient/outpatient), general physicians, palliative care services and the MS-registry of the German MS Society (DMSG) to preselect potentially eligible patients and their caregivers and refer them to the study office for further screening and recruitment. As soon as written permission is given, a trial physician will contact potential trial participants. The trial physician will decide on patient and caregiver eligibility. Patients might be enrolled into the trial even without a participating caregiver.

To keep the recruitment period-initially planned for 9 months-ongoing, study personnel, for example, the CCM, will regularly contact partner institutions/organisations and inquire about eligible patients.

\section{Eligibility criteria}

Detailed in-and exclusion criteria for patients in subgroup 1 (highly active patients with MS) and 2a, 2b (patients with primary or secondary chronic progressive MS) and their caregivers are provided in table 1 . If eligible, the trial physician will do the entire informed consent process (see online supplemental file 1 for the original consent form). The clinical project management or trial nurse will then coordinate all further steps, that is, baseline assessment by the blinded outcome assessor, randomisation and start of the CCM intervention. A flow chart of the intervention, follow-up period and visit schedule is given in figure 1 .

\section{Randomisation and blinding}

After the baseline assessment patients and their caregivers will be randomly assigned to either the intervention or the control group (allocation ratio 1:1). Randomisation will be done using a $24 / 7$ readily accessible internet-based tool (ALEA; FormsVision BV, Abcoude, Netherlands) and stratified by subgroup (1, 2a, 2b). We aim for an equal enrolment (1:1:1) in the three subgroups $(1,2 \mathrm{a}, 2 \mathrm{~b})$, but will accept an imbalance ratio of 2:1:1 considering the limited recruitment period of 9 months.

Patients will be informed by trial staff about their assignment via phone or mail. Only relevant information in order to get in contact with study participants will be provided to the independent outcome assessor without releasing information about treatment assignment as the outcome assessor is supposed to be blinded to treatment assignment throughout the entire trial. All study participants will regularly be reminded by study personnel not to disclose their group assignment to the outcome assessor.

\section{Intervention}

In the control group, patients with MS will receive standard care within existing healthcare structures. In the intervention group, patients will also receive standard care and will, in addition, regularly be contacted by one of two trained and certified CCMs with significant experience in patient-related care who will get paid according to the German wage agreement for the public service sector. The intervention comprises monthly personal visits by the CCM wherever the patient (and caregiver, if any) is (eg, at home) and weekly telephone calls ( \pm 3 days for both personal visits and telephone calls) (figure 1). Using the CCM manual (see online supplemental file 2 for our specifically developed CCM manual based on previous literature $\left.{ }^{22-34}\right)$, these visits will include assessment, goal setting, help planning, care coordination/collaboration, review of objectives and readjustment, where necessary, and documentation. ${ }^{23-25}$ 27-31 It will be used by all CCMs involved in the trial who have extensive experience with vulnerable patient populations. At the initial visit at baseline (T0), a thorough assessment will allow for development of a care plan focussing on priorities set by the patient and caregiver. Patient and caregiver will then commission the CCM to pursue set objectives. During the regular follow-ups (personal visits and telephone calls), the care plan will be continuously evaluated and adapted according to current problems and unmet needs, if necessary. This and undertaken measures will include meticulous documentation of identified needs, fragmented care, deficits in the healthcare system and advice for possible solutions given by the CCM which will be documented in the manual and reported back to involved physicians and healthcare providers, where needed. The CCM will be in close contact with the treating physician and healthcare services to ensure the coordination and monitoring of all relevant healthcare services necessary for each patient to ensure cross-sectoral transparent communication and an integrative level of care. Providers of appropriate sociomedical services identified for the care of study participants will be documented in a portfolio, which will be developed throughout the trial. Where needed, the CCM will arrange for new services to be provided by connecting existing structures, if possible. Otherwise, it will be documented as 'gap of care' in the portfolio that shall be addressed in the future, irrespective of this study. At the end of the intervention, the respective CCM will be released of its duties.

The burden of the intervention is kept to a minimum, as there are no foreseeable risks involved, except for a time investment. The intent of the CCMs is to be sensitive and respectful of the time constraints of the participants. 
Table 1 Inclusion and exclusion criteria of patients and caregivers

\section{Inclusion criteria patients}

\begin{tabular}{|c|c|c|}
\hline Subgroup 1 & \multicolumn{2}{|l|}{ Subgroup 2} \\
\hline $\begin{array}{l}\text { Highly active patients with MS, characterised } \\
\text { by relapses and MRI activity, with an indication } \\
\text { for an escalating immunotherapeutic agent } \\
\text { (eg, alemtuzumab, ocrelizumab, rituximab, } \\
\text { ofatumumab, natalizumab, fingolimod, siponimod, } \\
\text { ozanimod, cladribine) and are being treated with } \\
\text { such }\end{array}$ & \multicolumn{2}{|c|}{ Patients with primary or secondary chronic progressive MS } \\
\hline \multirow{4}{*}{$\begin{array}{l}\text { Age } \geq 50 \text { years } \\
\text { EDSS } \geq 5\end{array}$} & \multicolumn{2}{|l|}{ Age $\geq 18$ years } \\
\hline & \multicolumn{2}{|l|}{ AND either (a) or (b) } \\
\hline & & (b) \\
\hline & $\begin{array}{l}\text { Moderate disability (EDSS } 4-7 \text { ) } \\
\text { and no immunotherapeutic } \\
\text { treatment option (eg, due to lack } \\
\text { of disease activity in the form of } \\
\text { relapses or MRI) }\end{array}$ & $\begin{array}{l}\text { Severe disability (EDSS }>7 \text { ), with or } \\
\text { without immunotherapeutic treatment } \\
\text { options }\end{array}$ \\
\hline
\end{tabular}

\section{AND}

\section{Residence in the administrative district of Cologne}

AND one of the following (1) or (2)

1. Full command of German language skills (understanding, reading, responding) and ability to give written informed consent

2. If a patient is unable to give consent, a legal representative, who has a full command of the German language (understanding, reading, responding) and is able to give written informed consent can act on behalf of the patient

\section{Inclusion criteria caregivers}

Person primarily responsible for the patient's care

Age $\geq 18$ years

- Full command of the German language (understanding, reading, responding)

Ability to give written informed consent

\section{Exclusion criteria patients and caregivers}

Lack of consent to follow protocol

- Current drug abuse, alcohol abuse, or psychiatric illness, which, according to the trial physician, renders the patient or caregiver unfit for participation

- Any kind of dependency on the PI or sponsor (including being employed there)

- Legal incapacity (caregivers only)

Patients may participate in the trial irrespective of (1) the participation of their caregiver and (2) other secondary diagnoses (except those listed under exclusion criteria).

EDSS, extended disability status scale; MRI, magnetic resonance imaging; MS, multiple sclerosis; PI, principal investigator.

The method of data collection is structured to be as convenient as possible. The CCM manual has been linked to the emergent issues of the literature review and prior studies were considered in order to remain as minimally invasive to the patients' and caregivers' time as possible. Should a patient not be able to complete the items of the CCM manual a caregiver is allowed to act on behalf of the patient and answer instead (proxy assessment). To further reduce patient burden, the intervention will take place at the patients' and caregivers' whereabouts (eg, at home). Moreover, we hypothesise that our chosen patient group and their caregivers actually appreciate the regular visits by the CCM (and the outcome assessor) as this conveys interest in their problems and needs.

\section{Outcome measures}

Irrespective of group allocation, data will be collected with selected outcome measures in 3-month intervals wherever the study participant is (eg, at home): commencing at baseline prior to randomisation (T0), continuing throughout the 12-month intervention (T1-T4), concluding with the last follow-up after the intervention has ended (T5) (figure 1). To minimise the burden on the patient and caregiver, if any, all outcome assessments 


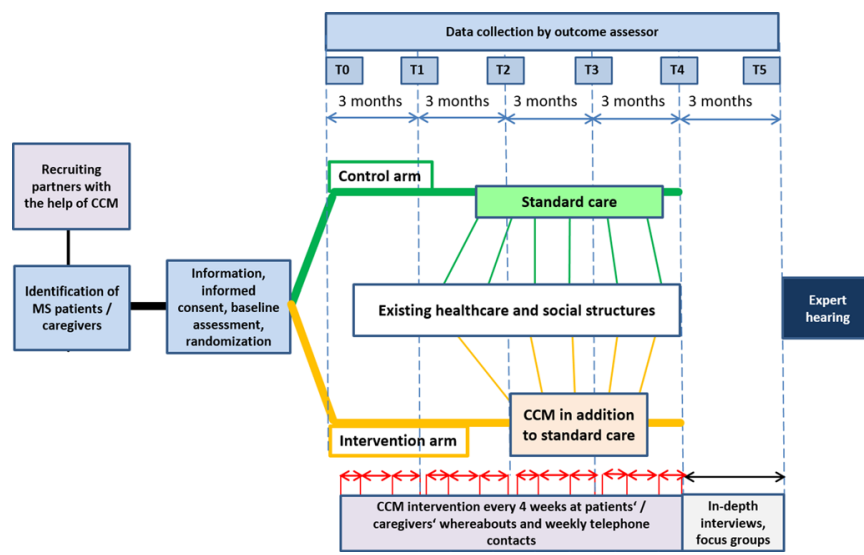

Figure 1 Trial flow chart. CCM, care and case management; MS, multiple sclerosis.

and quarterly CCM visits will be scheduled with a slight time lag of approximately 1 week apart from each other.

\section{QoL}

QoL will be measured with the 'Hamburger Lebensqualitätsmessinstrument' (HALEMS, German version of the Quality of Life Questionnaire for Multiple Sclerosis), which is responsive to change as shown in other intervention studies on patients with MS. ${ }^{35}$ The HALEMS can be requested from the author himself.

\section{Risk perception}

MS is characterised by many uncertainties including the risks of immunotherapeutic treatments. ${ }^{36}{ }^{37}$ For this trial, we have developed and pretested a questionnaire on risk perception for people with severe MS being treated with escalating immunotherapeutic agents following Heesen $e t$ $a l^{38}$ and Boeije $e t a l^{39}$ (online supplemental file 3). Using this questionnaire, patients will, inter alia, be asked for the reason of choosing a particular immunotherapeutic agent, their perceived severity of MS, to estimate the risks of their immunotherapeutic agent, and at which risk level they would stop treatment.

\section{Palliative care needs}

Patients with MS are seldomly cared for in palliative and hospice care structures, despite the growing evidence of the potential benefits thereof. ${ }^{50-43}$ A typical palliative care assessment tool is the (integrated) Palliative care Outcome Sale (POS/IPOS) ${ }^{44}{ }^{45}$ which has recently been extended by neurological items to evaluate the outcome for people with progressive, long-term neurological conditions, that is, the IPOS Neuro-S8. ${ }^{46}$ For this study, we will incorporate the IPOS Neuro-S8 into the IPOS. Both outcome measures can be downloaded from the POS website. As the IPOS Neuro-S8 has not yet been validated in the German language, we will additionally use the Hospice and Palliative Care Evaluation documentation supplemented by neurological symptoms for validation purposes, which also captures the comprehensive symptom burden of neurological patients in need of palliative care. ${ }^{47}$
Anxiety and depression

MS is a chronic progressive neurological disease with psychological and psychiatric implications. CM has been shown to address medical, financial, social and psychological problems of patients. ${ }^{7}$ The Hospital Anxiety and Depression Scale (HADS), ${ }^{48}$ for purchase at the Hogrefe Publishing Group, is a questionnaire developed to screen both anxiety and depression and is one of the most widely used questionnaires for patients with neurological conditions including $\mathrm{MS}^{49-51}$

\section{Caregivers' burden}

MS has a high burden on caregivers, ${ }^{52}$ which is influenced by a multitude of factors including the severity of the disease, type of disease, severity of symptoms and social support, all of which, can, in turn, affect QoL and mental and physical health of caregivers. ${ }^{53}$ The Zarit Burden Interview (ZBI) has been used to assess the burden of caregivers of patients with MS associated with functional or behavioural impairments and the home care situation and can be requested from the Mapi Research Trust organisation. $^{54}$

\section{Caregivers' QoL}

As mentioned above, MS affects the QoL of patients but also of their caregivers..$^{55}$ To minimise the time constraints on trial participants, we will use the 12-item Short Form Health Survey (SF-12), ${ }^{56}$ for purchase at the Hogrefe Publishing Group.

\section{Use of healthcare services}

Studies suggest that CM may lead to decreases in service use and healthcare costs. ${ }^{7}$ To evaluate this in our trial, we composed and pretested a structured cost booklet similar to the iMedical Consumption Questionnaire ${ }^{57}$ and Client Service Receipt Inventory ${ }^{58}$ (online supplemental file 4). The booklet addresses direct and indirect medical costs.

\section{Qualitative component}

Due to the delivery of the intervention, comprehensive interventions are difficult to evaluate and individual aspects are challenging to assess. Complex comprehensive interventions may involve unexpected aspects not planned for or not measurable by quantitative methods ${ }^{59}$; thus, knowledge from qualitative methods will aid in the interpretative process and evaluation of such interventions. Herein, the patients' perspective is of particular importance given the growing number of chronically ill patients and the significance of patient-centred care. The addition of the qualitative component may also allow to assess feasibility, acceptability and utility of the CCM intervention. As per recommendation for the evaluation of complex interventions, we will therefore apply a mixed-methods design. ${ }^{59}{ }^{60}$ This will involve individual in-depth interviews with 3-4 patients and caregivers of each subgroup as well as 5 focus groups with 3-12 healthcare professionals each (eg, neurologists, general practitioners, co-therapists, nursing staff). Both the interviews and focus groups will be conducted within the last 
3 months of the study. The interview guide has been developed by research staff in close cooperation with the principal investigator (PI) and CCM inspired by previous literature. ${ }^{22-34}$

\section{Sample size calculation}

For the primary outcome HALEMS, we assume an effect size of 0.5 to detect 'changes therein from baseline to month 12'. The HALEMS comprises 44 items that are scored on a 5/7-graded Likert scale and averaged thereafter. Gold $e t a l^{35}$ shows a group difference of 0.29 and 0.85 (average: 0.57 ) points between HADS-A and -D (i.e. $\leq 7 \mathrm{vs} \geq 8$ points). Note, that 7 points is the established threshold value for the HADS-A/-D to qualify for treatment. We will assume that $2 / 3$ of this averaged difference (ie, $0.57=(0.29+0.85) / 2)$ can be considered clinically relevant, which are 0.38 points. Consequently, 16-17 items (out of 44) will have to be scored higher or lower by one category. Assuming an SD of $0.74,{ }^{35}$ an effect size of 0.5 corresponds to the averaged difference of 0.38 point as derived above. Considering the baseline (T0) and four follow-up assessments (with a correlation of 0.5), 24 patients are required per treatment arm to detect a standardised effect of 0.5 with an $80 \%$ power at a two-sided significance level of 5\%, using linear Mixedeffect Model for Repeated Measurement (MMRM) ${ }^{61}$ The last follow-up (T5), 3 months after the intervention has ended, will be used as measure of sustainability. Accounting for up to $40 \%$ drop-out, $80 \quad(=2 * 24 / 0.6)$ patients need to be included and randomised. Thus, (the desired) equal enrolment (1:1:1) in the three subgroups (1, 2a, 2b) corresponds to 13-14 patients per subgroup and treatment arm.

\section{Data analysis plan}

The "change in the HALEMS from baseline to 12 months post-randomisation' is evaluated by a linear MMRM with fixed effects baseline, treatment arm, time, treatment arm*time, gender, underlying disease (heterogenous first-order autoregressive (ARH1)-structured covariance matrix over time) with corresponding marginal means and contrast tests (comparison of treatment arms after 12 months will be primary, after 15 months secondary). Since mixed models can be expected to yield valid results only in case of missingness-at-random, multiple imputation approaches are taken to assess the robustness of the results. Specifically, missing values due to death, illness, or chance are separately imputed assuming mixtures of missingness-not-at-random patterns. Imputation data sets are post-processed by multiplication with factors and addition of offsets (tipping point analysis).

Secondary outcomes (ie, further measures at the same time points) are analysed along the same lines. Timeto-event (eg, drop-out or survival) distributions are summarised by the Kaplan-Meier method and compared by the (stratified) log-rank test. Moreover, methods for competing events are applied.
The economic evaluation will be a cost-consequence analysis from the societal perspective, taking into account the implementation and running costs of the CCM. All costs incurred during the intervention will be determined using the cost booklet (online supplemental file 4). For the evaluation, it is of pivotal importance to determine and compare the costs and outcomes for the CCM intervention with the existing alternative (control group, ie, standard care) through disaggregated presentation of monetary consequences.

Qualitative data derived from individual in-depth interviews and focus groups will be analysed using a deductive qualitative content analysis. ${ }^{62}{ }^{63}$ Every transcribed interview will be read thoroughly to identify and code relevant text segments. Whenever relevant text segments cannot be classified, new subcodes will be created inductively. ${ }^{64}$ This will allow for an exploration of themes, which are of crucial importance for successful CCM.

\section{Patient and public involvement}

The patient association DMSG and its MS-Registry have been involved in this trial since the development of the study design, including the study population' definition. During the trial, they are mainly engaged in patient preselection for subsequent recruitment into the trial and will provide their networks and communities for dissemination of trial results.

\section{ETHICS AND DISSEMINATION \\ Ethical considerations}

The trial protocol and any amendments have been and will be prepared in accordance with the principles of the Declaration of Helsinki. Changes to the trial protocol may only be implemented if agreed by the sponsor and all authors of the trial protocol and after favourable opinion of the ethics committee. The trial was approved by the Ethics Commission of Cologne University's Faculty of Medicine (\#20-1436). All trial subjects will be informed that participation is fully voluntary and that refusal to participate or discontinuation of participation at any time will be without any disadvantages or loss of benefits to which the subject is otherwise entitled.

\section{Monitoring}

The trial site will be monitored by the Clinical Trials Centre Cologne (CTCC) to ensure the quality of the data collected. The objectives of the monitoring procedures are to ensure that the trial subject's safety and rights as a trial participant are respected, that accurate, valid, and complete data are collected and that the trial is conducted in accordance with the trial protocol, the principles of Good Clinical Practice (GCP), and local legislation.

\section{Documentation}

All data relevant to the trial, if not directly documented in the Electronic Case Report Form (eCRF, see below), are entered into the eCRF timely after being obtained by 
the responsible trial staff member. The outcome assessor will enter the data remotely into the database (eCRF) during data collection at the patients' and caregivers' home using a laptop. This includes all outcome measures, sociodemographic (see online supplemental file 5) and compliance data (see online supplemental file 6). In addition, the outcome assessor will always have paperand-pencil versions (interim CRF) at hand and can thus complete these in the event of, for example, technical failure but transfer the data into the eCRFs as soon as possible. The database has been sufficiently validated and tested. Every correction or alteration is traceable. Only authorised people will have access to the programme and all data. Regular data backups will be made. The eCRFs will be signed by the PI.

The eCRF provides specific sections where data have to be documented. All site staff involved in the documentation of data will receive individualised login data. The PI is responsible for assuring that the data entered into eCRF is complete, accurate, and that data entry and updates are performed in a timely manner. During the trial, the PI may authorise individual trial staff members to sign the eCRFs to confirm data accuracy. At the end of the follow-up visits, the PI must sign the eCRF.

\section{Data management}

The information technology (IT) infrastructure and data management staff will be supplied by the CTCC. The trial database will be developed and validated before data entry based on standard operating procedures (SOPs) at the CTCC. The data management system is based on commercial trial software and stores the data in a database. All changes made to the data are documented in an audit trail. The trial software has a user and role concept that can be adjusted on a trial-specific basis. The database is integrated into a general IT infrastructure and safety concept with a firewall and backup system. The data are backed up daily. After completion and cleaning of data, the database is locked and the data exported for statistical analysis.

The data will be entered online. Plausibility checks are run during data entry, thereby detecting discrepancies immediately. The CTCC Data Management will conduct further checks for completeness and plausibility and will clarify any questions according to existing SOPs via queries. These electronic queries have to be answered by trial staff members without unreasonable delay.

\section{Data protection}

The provisions of data protection legislation will be observed. It is assured by the sponsor that all investigational materials and data will be pseudonymised in accordance with data protection legislation before scientific analyses.

Trial subjects will be informed that their pseudonymised data will be passed on in agreement with provisions for documentation and notification pursuant to GCP regulations and handled in accordance with applicable law.
Subjects who do not agree to data handling as described in the informed consent form will not be enrolled into the trial. Procedures for potential auditing trial conduct are described in the informed consent form as well.

During the trial, all trial data will be captured in a validated database. The database format and structure allow for long-term maintenance and accessibility. To ensure this, all data for long-term accessibility will be archived in anonymised form using standard data formats.

\section{Safety considerations}

We do not expect any adverse events in this non-"Arzneimittelgesetz" / non-"Medizinproduktegesetz" (non-AMG/ non-MPG) clinical trial. Worsening of the patients' general health condition or perhaps even death, depending on the severity of MS, throughout the intervention and follow-up is not unusual. CM intervention has been shown to be beneficial for chronic neurological and other patients. ${ }^{7-10} 12$ 16-20 22 65-67 In both the intervention and comparator group, patients will have access to all existing healthcare structures. Potential distress phenomena will be identified by the outcome assessor during the quarterly personal visits using our chosen outcome measures (HALEMS, ${ }^{35}$ IPOS/IPOS NeuroS8, ${ }^{44-46}$ HADS, $^{48}$ ZBI-12, ${ }^{68-70}$ SF-12 ${ }^{56}$ as they are, inter alia, capturing physical (pain, constipation, spasms, etc), psychological (anxiety, depression, etc) and relational (family, friends, etc) aspects. In the event of a conspicuous value on any of these outcome measures indicating high level of distress, they will be reported back to the PI, irrespective of group allocation. The PI will notify the primary treating physician who will then decide on how to proceed (eg, intervene personally, involve other professional healthcare services, or terminate the trial for this particular subject). We are therewith ensuring a prompt and competent approach in the unlikely event of potential distress. The follow-up ( 3 months after the end of the intervention) allows for evaluation of sustainability but also to continue assessing potential distress. The PI will keep an updated list, categorise all potential distress phenomena, and discuss those with the co-investigators and all involved staff midtrial unless the PI sees need for an earlier meeting. Due to the current COVID-19 pandemic, we have developed a comprehensive hygiene concept.

\section{Dissemination plan}

Our final results will be presented and discussed with relevant healthcare experts in an expert hearing and reported back to study participants. Trial results will be published in peer-reviewed scientific journals and presented at national and international conferences.

\section{Strengths and limitations}

This is the first trial to evaluate the effects of a crosssectorial, outreaching, long-term patient advocacy CCM for severely affected patients with MS with complex needs 
and their caregivers over a period of 15 months including one follow-up assessment.

Our study has several strengths. First, a follow-up assessment allows for the evaluation of sustainability and assessment of potential aftereffects. Second, as we assume that CCM is especially useful in patients with complex neurological disease, we selected patients with a higher degree of MS-related disability and chronic progressive disease, higher risk for treatment-related adverse effects or higher age. All of these factors might negatively impact the benefit-risk ratio of modern immunotherapy. Our selected patient population can therefore be considered a pioneer population of a complex neurological disease. Third, our study design has been developed to find evidence for the effectiveness of CCM. In close collaboration with the statistician $(\mathrm{MH})$, we have developed a powerful design, which is evident from the selection of patients, randomisation, permission of proxy assessments, blinding of the outcome assessor, selection of reliable and validated outcome measures and the statistical methods. With our chosen mixed methodology we might gain more specific insight into the perspectives of study participants in the intervention group within our quantitative study and get a fuller, more complex picture.

However, there are also a number of caveats that relate specifically to the methods. First, while permission of proxy assessments will reduce missing data, this might potentially introduce bias and should therefore be interpreted with caution. Second, blinding of the outcome assessor depends on the discretion of study participants, which might not always be feasible. In the event of unintended unblinding, the outcome assessor will document this into the eCRF by means of the compliance form so this can be taken into consideration when analysing the data. Last, there are potential limitations which could impact our design and analytic plan. Specifically, the planned sample size (80 patients in total) in three strata (13-14 per stratum and treatment arm) may be too small to sufficiently estimate all relevant aspects of the proposed model for longitudinal data, particularly in all subgroups of interest.

\section{Author affiliations}

${ }^{1}$ Department of Palliative Medicine, University of Cologne, Cologne, Germany

${ }^{2}$ Institute of Medical Statistics and Computational Biology (IMSB), University of Cologne, Cologne, Germany

${ }^{3}$ Clinical Trials Centre Cologne (CTCC), University of Cologne, Cologne, Germany ${ }^{4}$ Institute for Health Economics and Clinical Epidemiology (IGKE), University of Cologne, Cologne, Germany

${ }^{5}$ Department of Neurology, University of Cologne, Cologne, Nordrhein-Westfalen, Germany

${ }^{6}$ Cognitive Neuroscience, Institute of Neurosciences and Medicine, Jülich, Germany

${ }^{7}$ Center for Integrated Oncology Aachen Bonn Cologne Düsseldorf (CIO ABCD),

University of Cologne, Cologne, Germany

${ }^{8}$ Center for Health Services Research (ZVFK), University of Cologne, Cologne, Germany

${ }^{9}$ MS-Registry by the German MS-Society, MS Forschungs- und

Projektentwicklungs-gGmbH, Hannover, Germany

${ }^{10}$ German Society for Care and Case Management (DGCC), Mainz, Germany

${ }^{11}$ Department of Neurology, Heilig Geist-Krankenhaus Köln, Cologne, Germany

${ }^{12}$ Department of Neurology, Klinikum Köln, Cologne, Germany
${ }^{13}$ NeuroMed Campus, MedCampus Hohenlind, Cologne, Germany

${ }^{14}$ Academic Teaching Practice, University of Cologne, Cologne, Germany

${ }^{15}$ German Multiple Sclerosis Society Federal Association (DMSG), Hannover, Germany

Acknowledgements We thank Dirk Müller for his valuable support and advice on the economic evaluation. The Clinical Trials Centre Cologne (CTCC) is responsible for performing non-clinical project management as well as tasks including project management, monitoring and data management.

Contributors $\mathrm{HG}, \mathrm{CW}$ and $\mathrm{MH}$ conceived and designed the trial. $\mathrm{HG}, \mathrm{KD}$ and $\mathrm{MH}$ wrote the draft trial protocol; herein $\mathrm{MH}$ wrote the statistical methods. VM designed the cost booklet. SU, TD and HG designed the CCM manual. CW, YG, KD, VD and $\mathrm{HG}$ designed the risk perception questionnaire. PS and AS contributed to the description of all ethical and regulatory aspects as well as data quality assurance. MH, TD, PS, AS, VD, JS, RV, SS, CW, YG, SU, VM, GRF, OC, AM, AS, PL, LB, VL, EB, KG, $\mathrm{GN}, \mathrm{TJ}, \mathrm{JH}$ and $\mathrm{HT}$ reviewed and commented drafts of the manuscript. All authors read and approved the final manuscript.

Funding This work was supported by the Innovation Funds of the Federal Joint Committee (G-BA), grant number 01VSF19029.

Competing interests None declared.

Patient consent for publication Not applicable.

Provenance and peer review Not commissioned; externally peer reviewed.

Supplemental material This content has been supplied by the author(s). It has not been vetted by BMJ Publishing Group Limited (BMJ) and may not have been peer-reviewed. Any opinions or recommendations discussed are solely those of the author(s) and are not endorsed by BMJ. BMJ disclaims all liability and responsibility arising from any reliance placed on the content. Where the content includes any translated material, BMJ does not warrant the accuracy and reliability of the translations (including but not limited to local regulations, clinical guidelines, terminology, drug names and drug dosages), and is not responsible for any error and/or omissions arising from translation and adaptation or otherwise.

Open access This is an open access article distributed in accordance with the Creative Commons Attribution Non Commercial (CC BY-NC 4.0) license, which permits others to distribute, remix, adapt, build upon this work non-commercially, and license their derivative works on different terms, provided the original work is properly cited, appropriate credit is given, any changes made indicated, and the use is non-commercial. See: http://creativecommons.org/licenses/by-nc/4.0/.

\section{ORCID iDs}

Heidrun Golla http://orcid.org/0000-0002-4403-630X

Julia Strupp http://orcid.org/0000-0003-3135-2693

Clemens Warnke http://orcid.org/0000-0002-3510-9255

\section{REFERENCES}

1 Strupp J, Voltz R, Golla H. Opening locked doors: integrating a palliative care approach into the management of patients with severe multiple sclerosis. Mult Scler 2016;22:13-18.

2 Galushko M, Golla H, Strupp J, et al. Unmet needs of patients feeling severely affected by multiple sclerosis in Germany: a qualitative study. J Palliat Med 2014;17:274-81.

3 Borreani C, Bianchi E, Pietrolongo E, et al. Unmet needs of people with severe multiple sclerosis and their carers: qualitative findings for a home-based intervention. PLoS One 2014;9:e109679.

4 Golla H, Mammeas S, Galushko M, et al. Unmet needs of caregivers of severely affected multiple sclerosis patients: a qualitative study. Palliat Support Care 2015;13:1685-93.

5 Golla H, Galushko M, Pfaff H, et al. Unmet needs of severely affected multiple sclerosis patients: the health professionals' view. Palliat Med 2012;26:139-51.

6 Halper J, MSCN APN-C. Advanced concepts in multiple sclerosis nursing care. Demos Medical Publishing, 2007.

7 Oeseburg B, Wynia K, Middel B, et al. Effects of case management for frail older people or those with chronic illness: a systematic review. Nurs Res 2009;58:201-10.

8 Basu A, Kee R, Buchanan D, et al. Comparative cost analysis of housing and case management program for chronically ill homeless adults compared to usual care. Health Serv Res 2012;47:523-43.

9 Chouinard M-C, Hudon C, Dubois M-F, et al. Case management and self-management support for frequent users with chronic disease in 
primary care: a pragmatic randomized controlled trial. $B M C$ Health Serv Res 2013;13:49.

10 Meier DE, Thar W, Jordan A, et al. Integrating case management and palliative care. J Palliat Med 2004;7:119-34.

11 Aiken LS, Butner J, Lockhart CA, et al. Outcome evaluation of a randomized trial of the PhoenixCare intervention: program of case management and coordinated care for the seriously chronically ill. $J$ Palliat Med 2006;9:111-26.

12 Casarin SNA, Villa TCS, Gonzáles RIC, et al. Case management: evolution of the concept in the 80's and 90's. Rev Lat Am Enfermagem 2002;10:472-7.

13 Kuhn U, Düsterdiek A, Galushko M, et al. Identifying patients suitable for palliative care--a descriptive analysis of enquiries using a Case Management Process Model approach. BMC Res Notes 2012;5:611.

14 Wynia K, Annema C, Nissen H, et al. Design of a randomised controlled trial (RCT) on the effectiveness of a Dutch patient advocacy case management intervention among severely disabled multiple sclerosis patients. BMC Health Serv Res 2010;10:142.

15 Strupp J, Dose C, Kuhn U, et al. Analysing the impact of a case management model on the specialised palliative care multiprofessional team. Support Care Cancer 2018;26:673-9.

16 Van Mierlo LD, Meiland FJM, Van de Ven PM, et al. Evaluation of DEM-DISC, customized e-advice on health and social support services for informal carers and case managers of people with dementia; a cluster randomized trial. Int Psychogeriatr 2015;27:1365-78.

17 Lukas A, Kilian R, Hay B, et al. [Maintenance of health and relief for caregivers of elderly with dementia by using "initial case management": experiences from the Lighthouse Project on Dementia, UIm, ULTDEM-study]. Z Gerontol Geriatr 2012:45:298-309.

18 Stergiopoulos V, Gozdzik A, Misir V, et al. Effectiveness of housing first with intensive case management in an ethnically diverse sample of homeless adults with mental illness: a randomized controlled trial. PLoS One 2015;10:e0130281.

19 Kivelitz L, Schulz H, Melchior H, et al. Effectiveness of case management-based aftercare coordination by phone for patients with depressive and anxiety disorders: study protocol for a randomized controlled trial. BMC Psychiatry 2015;15:1-7.

20 Meisinger C, Stollenwerk B, Kirchberger I, et al. Effects of a nursebased case management compared to usual care among aged patients with myocardial infarction: results from the randomized controlled KORINNA study. BMC Geriatr 2013;13:1-10.

21 Chan A-W, Tetzlaff JM, Gøtzsche PC, et al. SPIRIT 2013 explanation and elaboration: guidance for protocols of clinical trials. BMJ 2013;346:e7586.

22 Ewers M, Schaeffer D. Case management in Theorie und praxis. Bern: Huber, 2005.

23 Wissert M. Tools und Werkzeuge beim case management: Outreaching-Case Finding-Intaking: Aufgaben Der Fallsteuerung und Der Systemsteuerung. Case Management 2006;2006:43-6.

24 Wissert M. Tools und Werkzeuge beim case management: Das assessment. Case Management 2006;2:107-8.

25 Wissert M, Vogt A, Radbruch L. Wirkungen von Case Management-Qualifizierung, Ergebnisse eines Modellprojekts zum "Unterstützungsmanagement in der palliativen und hospizlichen Arbeit". Case Management 2009;6:36-44.

26 Wendt WR. Case management: ein Konzept. viele Anwendungen 2005;2:7-9.

27 Wissert M. Tools und Werkzeuge beim case management: die Hilfeplanung. Case Management 2007;1:35-7.

28 Wissert M. Tools und Werkzeuge beim case management: linking: Aufgaben Der Fallsteuerung und Der Systemsteuerung. Case Management 2007;2:82-4.

29 Wissert M. Tools und Werkzeuge beim case management monitoring: Aufgaben Der Fallsteuerung und Der Systemsteuerung Case Management 2008;1:33-5.

30 Wissert M. Tools und Werkzeuge beim case management: evaluation und Entpflichtung. Case Management 2008;2:98-100.

31 Wissert M. Tools und Werkzeuge beim case management: Evaluierende Nachsorge. Case Management 2009;1:34-5.

32 Wissert M, Roccor B. Case management in palliative CareKontexten. Case Management 2013;1:16-23.

33 Institut für Praxisforschung und Evaluation. Projektbericht: evaluation des case managements: Interviewphase, 2017. Available: https://da.rummelsberger-diakonie.de/fileadmin/user upload/WI/Evaluation_Case-Management_2017.pdf [Accessed 26 May 2020].

34 National Institute for Health and Care Excellence. Multiple sclerosis in adults: management. Available: https://www.nice.org.uk/guidance/ cg186 [Accessed 26 May 2020].
35 Gold SM, Heesen C, Schulz H, et al. Disease specific quality of life instruments in multiple sclerosis: validation of the Hamburg quality of life questionnaire in multiple sclerosis (HAQUAMS). Mult Scler 2001;7:119-30.

36 Weideman AM, Tapia-Maltos MA, Johnson K, et al. Meta-Analysis of the age-dependent efficacy of multiple sclerosis treatments. Front Neurol 2017;8:577.

37 Schweitzer F, Laurent S, Fink GR, et al. Age and the risks of highefficacy disease modifying drugs in multiple sclerosis. Curr Opin Neurol 2019;32:305-12.

38 Heesen C, Kleiter I, Nguyen F, et al. Risk perception in natalizumabtreated multiple sclerosis patients and their neurologists. Mult Scler 2010;16:1507-12.

39 Boeije HR, Janssens ACJW. 'It might happen or it might not': how patients with multiple sclerosis explain their perception of prognostic risk. Soc Sci Med 2004;59:861-8.

40 Edmonds $\mathrm{P}$, Hart S, et al. Palliative care for people severely affected by multiple sclerosis: evaluation of a novel palliative care service. Mult Scler 2010;16:627-36.

41 Wollin JA, Yates PM, Kristianson LJ. Supportive and palliative care needs identified by multiple sclerosis patients and their families. Int $J$ Palliat Nurs 2006;12:20-6.

42 Ben-Zacharia AB, Lublin FD. Palliative care in patients with multiple sclerosis. Neurol Clin 2001;19:801-27.

43 Voltz R. Palliative care for multiple sclerosis: a counter-intuitive approach? Mult Scler 2010;16:515-7.

44 Bausewein C, Le Grice C, Simon S, et al. The use of two common palliative outcome measures in clinical care and research: a systematic review of Pos and STAS. Palliat Med 2011;25:304-13.

45 Sleeman KE, Higginson IJ. A psychometric validation of two brief measures to assess palliative need in patients severely affected by multiple sclerosis. J Pain Symptom Manage 2013;46:406-12.

46 Gao W, Crosby V, Wilcock A, et al. Psychometric properties of a generic, patient-centred palliative care outcome measure of symptom burden for people with progressive long term neurological conditions. PLoS One 2016;11:e0165379.

47 Dillen K, Ebke M, Koch A, et al. Validation of a palliative care outcome measurement tool supplemented by neurological symptoms (HOPE+): identification of palliative concerns of neurological patients. Palliat Med 2019;33:1221-31.

48 Bjelland I, Dahl AA, Haug TT, et al. The validity of the hospital anxiety and depression scale. An updated literature review. J Psychosom Res 2002;52:69-77.

49 Pais-Ribeiro JL, Martins da Silva A, Vilhena E, et al. The hospital anxiety and depression scale, in patients with multiple sclerosis. Neuropsychiatr Dis Treat 2018;14:3193.

50 Watson TM, Ford E, Worthington E, et al. Validation of mood measures for people with multiple sclerosis. Int J MS Care 2014:16:105-9.

51 Hind D, Kaklamanou D, Beever D, et al. The assessment of depression in people with multiple sclerosis: a systematic review of psychometric validation studies. BMC Psychiatry 2016;16:278.

52 Petrikis P, Baldouma A, Katsanos AH, et al. Quality of life and emotional strain in caregivers of patients with multiple sclerosis. $J$ Clin Neurol 2019;15:77-83.

53 Alshubaili AF, Ohaeri JU, Awadalla AW, et al. Family caregiver quality of life in multiple sclerosis among Kuwaitis: a controlled study. BMC Health Serv Res 2008;8:206.

54 Algahtani $\mathrm{H}$, Shirah B, Bayazeed A, et al. Assessment of the burden of multiple sclerosis patients' caregivers in Saudi Arabia. Cureus 2020;12:e6658

55 McKeown LP, Porter-Armstrong AP, Baxter GD, . The needs and experiences of caregivers of individuals with multiple sclerosis: a systematic review. Clin Rehabil 2003;17:234-48.

56 Salyers MP, Bosworth HB, Swanson JW, et al. Reliability and validity of the SF-12 health survey among people with severe mental illness. Med Care 2000;38:1141-50.

57 et alBouwmans C, Hakkaart-van Roijen L, Koopmanschap M. iMTA medical consumption questionnaire Rotterdam, 2013. Available: https://www.imta.nl/questionnaires/ [Accessed 2 May 2020].

58 Thornicroft GE, Brewin CR, Wing JE. Measuring mental health needs. London: Gaskell/Royal College of Psychiatrists, 1992.

59 Craig P, Dieppe P, Macintyre S, et al. Developing and evaluating complex interventions: the new medical Research Council guidance. Int J Nurs Stud 2013:50:587-92.

60 Higginson IJ, Evans CJ, Grande G, et al. Evaluating complex interventions in end of life care: the MORECare statement on good practice generated by a synthesis of transparent expert consultations and systematic reviews. BMC Med 2013;11:111.

61 Machin D, Campbell M, Tan S. Sample size tables for clinical studies. Chichester, West Sussex, UK: Wiley-Blackwell, 2011. 
62 Straus A, Corbin J. Basics of qualitative research: Grounded theory procedures and techniques. Newbury Park, CA: Sage, 1990.

63 Mayring P. Qualitative Inhaltsanalyse. Grundlagen und Techniken. 12. überarbeitete Auflage. Weinheim: Beltz, 2015.

64 Kuckartz U. Einführung in die computergestützte analyse qualitativer Daten, 3., aktualisierte Auflage. Wiesbaden: VS Verlag für Sozialwissenschaften, 2010.

65 Neuffer M. Case management: Soziale Arbeit MIT Einzelnen und Familien. Weinheim Basel: Beltz Juventa, 2002.

66 McAlister FA, Grover S, Padwal RS, et al. Case management reduces global vascular risk after stroke: secondary results from the the preventing recurrent vascular events and neurological worsening through intensive organized case-management randomized controlled trial. Am Heart J 2014;168:924-30.

67 Kirchberger I, Hunger M, Stollenwerk B, et al. Effects of a 3year nurse-based case management in aged patients with acute myocardial infarction on rehospitalisation, mortality, risk factors, physical functioning and mental health. A secondary analysis of the randomized controlled KORINNA study. PLoS One 2015;10:e0116693.

68 Zarit SH, Reever KE, Bach-Peterson J. Relatives of the impaired elderly: correlates of feelings of burden. Gerontologist 1980;20:649-55.

69 Bédard M, Molloy DW, Squire L, et al. The Zarit burden interview: a new short version and screening version. Gerontologist 2001;41:652-7.

70 Braun M, Scholz U, Hornung R, et al. [Caregiver burden with dementia patients. A validation study of the German language version of the Zarit Burden Interview]. Z Gerontol Geriatr 2010;43:111-9. 